\title{
Influence of handrail use of kinematic effects of ascending and descending stair in old and young age
}

\author{
Byung-Chan Min ${ }^{1}$, Jin-Whan Kwon ${ }^{1}$, Beum-Soo Lee ${ }^{1}$, Young-Sun Kim ${ }^{1}$, Jin-Kyu Kang \\ ${ }^{1}$ Department of Industrial \& Management Engineering, \\ Hanbat National University, Daejon, Korea \\ Corresponding author's e-mail: $\underline{\text { bcmin @, hanbat.ac.kr }}$
}

\begin{abstract}
The present study aimed to examine the influence of handrail use of the kinematic effects of ascending and descending stair in old and young age. A total of thirty-two subjects ascended and descended a purpose-built stair. This experiment conducted to three conditions within no hand, one hand and both hand as use handrails and analyzed gait characteristics. During stair up-down the elderly and young adults generated similar joint movement. However, the difference that noted at hip-rotation between elderly and young adults in case of both hands and one handle use on up-stairs.
\end{abstract}

Keywords: stairs, older adults, handrails, kinematic effects

\section{Introduction}

Stairs are frequently encountered obstacles in daily living. Although healthy persons climb stairs quite easily, this movement task is demanding when motor functions are reduced, for example in elderly. It has been reported the joint kinematic during stair descent are similar between young and older adults (Hortiobagyi et al., 2003; Mian et al., 2007). However, the elderly may be operating closer to their maximal joint range of motion limits than young adults. It seems to hypothesize that handrail use will improve safety for older adults on stairs. We hypothesized that use of handrails in older adults would aid dynamic balance control during ascending and descending stairs. This study was to compare lower extremity motion during stair descent and ascend in young and older adults. We focused on descent because it is more challenging than ascent with handrails.

\section{Methods}

\subsection{Participants}

Thirty-two healthy subjects, 16 older adults and 16 young persons were tested in this study. None of the subjects reported any neurological, sensor motor or musculoskeletal disorder or deficits, or use of drugs that might affect postural balance, ability to ascend or descend a stairway or ability to grasp a handrail (table 1).

Table 1.

mean \pm S.D. physical characteristics of older and young

\begin{tabular}{ccc}
\hline & Old adults & Young adults \\
\cline { 2 - 3 } & Mean SD & Mean SD \\
\hline Age(years) & $71.39 \pm 3.5$ & $23.6 \pm 2.6$ \\
\hline Height(cm) & $167.38 \pm 4.319$ & $176.5 \pm 6.2$ \\
\hline Mass(kg) & $65 \pm 4.648$ & $71.4 \pm 8.8$ \\
\hline Right Length(nm) & $238.81 \pm 10.329$ & $257.8 \pm 13.2$ \\
foot Width(nm) & $91.63 \pm 4.241$ & $98.2 \pm 4.3$ \\
\hline
\end{tabular}

2.2 experimental equipment.

Stairs used to this study were designed to the structure that a handrail was installed as purpose-built stairs . Eight cameras which were arranged around stairs in order to photograph an action of stairs walk were mobilized. A concrete characteristic of stairs and experiment equipment is same as the following (table 2).

Table 2. experimental equipment

\begin{tabular}{|c|c|c|}
\hline Distribution & item. & Contents. \\
\hline \multirow{6}{*}{ Variable stairs. } & High of a stair & $20 \mathrm{~cm}$ \\
\hline & Width of a stair & $28 \mathrm{~cm}$ \\
\hline & $\begin{array}{c}\text { Right and left width } \\
\text { of a stair }\end{array}$ & $81 \mathrm{~cm}$ \\
\hline & A number of a stair & 6 \\
\hline & High of a handle. & $85 \mathrm{~cm}$ \\
\hline & Handle diameter. & $3.5 \mathrm{~cm}$ \\
\hline Distribution. & Appliances name. & Manufacture \\
\hline $\begin{array}{l}\text { Photograph } \\
\text { equipment }\end{array}$ & Hawk. & \multirow{3}{*}{$\begin{array}{l}\text { Motion. } \\
\text { Analysis. } \\
\text { (USA). }\end{array}$} \\
\hline \multirow{2}{*}{ Analysis software. } & EVaRT 5.04 & \\
\hline & OrthoTrak 6.5.1. & \\
\hline
\end{tabular}

\section{3 experimental procedure and analysis method}

Participants negotiated a purpose-built of 4-step instrumented staircase. A eight-camera motion analysis system (EVaRT 5.0 Motion analysis, USA) sampling at $60 \mathrm{~Hz}$ was used to capture the displacement of reflective markers placed on specific anatomical landmarks of according to the guidelines of the Helen Hayes marker set placement(table 2). This experiment accomplished at three conditions regarding stairs handle. This experiment conducted to three conditions within no hand, one hand, and both hand as 
use handrails. Comparisons between Young and old were made using a paired t-test. Data are presented as mean and standard deviation.

\section{Results}

3.1 Joint angle difference in handrail use of descending and ascending stairs

Average joint angle data are shown in table 3. In frontal plane, no differences were observed in pelvic up/down obliquity, hip ab/adduction, or knee varus valgus and foot progression (table 3). .In the sagittal plane, no differences were observed in dorsiplantarflexion, knee flex-extension, hip flex-extension and pelvic tilt. Age-related difference was present in the transverse plane. Internal hip rotation in late stance was greater in older $(\mathrm{t}=3.179, \mathrm{p}<0.05)$.

Table 3

mean \pm SD joint angles(in degree) in young and older

\begin{tabular}{|c|c|c|c|c|}
\hline \multicolumn{3}{|c|}{ Frontal plane } & \multicolumn{2}{|c|}{ Movement } \\
\hline & handrail & Age & Ascending & Descending \\
\hline \multirow{3}{*}{$\begin{array}{l}\text { Foot } \\
\text { pro. }\end{array}$} & No-one & $\begin{array}{l}\text { Young } \\
\text { Older }\end{array}$ & $\begin{array}{l}.3 .057 \pm 30.133 \\
10.519 \pm 35.208\end{array}$ & $\begin{array}{l}8.01 \pm 59.984 \\
68.973 \pm 226.017\end{array}$ \\
\hline & $\begin{array}{l}\text { One } \\
\text {-both }\end{array}$ & $\begin{array}{l}\text { Young } \\
\text { Older }\end{array}$ & $\begin{array}{l}-6.815 \pm 24.503 \\
-.536 \pm 33.022\end{array}$ & $\begin{array}{l}8.671 \pm 38.719 \\
-11.148 \pm 32.809\end{array}$ \\
\hline & $\begin{array}{l}\begin{array}{l}\text { No } \\
\text {-both }\end{array} \\
\end{array}$ & $\begin{array}{l}\text { Young } \\
\text { Older }\end{array}$ & $\begin{array}{l}2.132 \pm 24.253 \\
-3.211 \pm 17.134 \\
\end{array}$ & $\begin{array}{l}-9621 \pm 53.889 \\
-1.647 \pm 10.673 \\
\end{array}$ \\
\hline \multirow[t]{3}{*}{$\begin{array}{l}\text { Knee } \\
\text { Vv }\end{array}$} & No-one & $\begin{array}{l}\text { Young } \\
\text { Older }\end{array}$ & $\begin{array}{l}9743 \pm 12.6448 \\
-16.611 \pm 48.029\end{array}$ & $\begin{array}{l}5.51 \pm 30.128 \\
11.349 \pm 37.34\end{array}$ \\
\hline & $\begin{array}{l}\begin{array}{c}\text { One } \\
\text {-both }\end{array} \\
\end{array}$ & $\begin{array}{l}\text { Young } \\
\text { Older }\end{array}$ & $\begin{array}{l}7.144 \pm 29.97 \\
7.778 \pm 14.352\end{array}$ & $\begin{array}{l}-.016 \pm 15.200 \\
.515 \pm 13.17\end{array}$ \\
\hline & $\begin{array}{l}\begin{array}{l}\text { No } \\
\text {-both }\end{array} \\
\end{array}$ & $\begin{array}{l}\text { Young } \\
\text { Older }\end{array}$ & $\begin{array}{l}-2.943 \pm 19.124 \\
-7.024 \pm 12.61 \\
\end{array}$ & $\begin{array}{l}4.191 \pm 29.335 \\
10.697 \pm 37.527 \\
\end{array}$ \\
\hline \multirow[t]{3}{*}{$\begin{array}{l}\text { Hip } \\
\text { Add. }\end{array}$} & No-one & $\begin{array}{l}\text { Young } \\
\text { Older }\end{array}$ & $\begin{array}{l}2.150 \pm 12.009 \\
.568 . \pm 24.397\end{array}$ & $\begin{array}{l}-2.293 \pm 18.886 \\
-.365 \pm 20.132\end{array}$ \\
\hline & $\begin{array}{l}\text { One } \\
\text {-both }\end{array}$ & $\begin{array}{l}\text { Young } \\
\text { Older }\end{array}$ & $\begin{array}{l}-.843 \pm 13.660 \\
-9.609 \pm 14.298\end{array}$ & $\begin{array}{l}-.489 \pm 23.067 \\
-8.231 \pm 18.075\end{array}$ \\
\hline & $\begin{array}{l}\text { No } \\
\text {-both }\end{array}$ & $\begin{array}{l}\text { Young } \\
\text { Older }\end{array}$ & $\begin{array}{l}2.246 \pm 22.34 \\
-1.012 \pm 15.862\end{array}$ & $\begin{array}{l}-3.604 \pm 26.336 \\
-10.766 \pm 16.131\end{array}$ \\
\hline \multirow[t]{3}{*}{$\begin{array}{l}\text { Pelvic } \\
\text { Obl. }\end{array}$} & No-one & $\begin{array}{l}\text { Young } \\
\text { Older }\end{array}$ & $\begin{array}{l}3.272 \pm 21.063 \\
-4.707 \pm 10.847\end{array}$ & $\begin{array}{l}-8.291 \pm 18.886 \\
-.354 \pm 26.042\end{array}$ \\
\hline & $\begin{array}{l}\text { One } \\
\text {-both }\end{array}$ & $\begin{array}{l}\text { Young } \\
\text { Older }\end{array}$ & $\begin{array}{l}-4.617 \pm 14.798 \\
-2.209 \pm 9.97\end{array}$ & $\begin{array}{l}-3.307 \pm 18.013 \\
-.992 \pm 21.35\end{array}$ \\
\hline & $\begin{array}{l}\text { No } \\
\text {-both }\end{array}$ & $\begin{array}{l}\text { Young } \\
\text { Older }\end{array}$ & $\begin{array}{l}-.850 \pm 6.995 \\
109.976 \pm 444.617\end{array}$ & $\begin{array}{r}2.558 \pm 23.919 \\
-3.173 \pm 25.977\end{array}$ \\
\hline \multicolumn{3}{|c|}{ Sagittal plane } & \multicolumn{2}{|c|}{ Movement } \\
\hline & Handrail & Age & Ascending & Descending \\
\hline \multirow{3}{*}{$\begin{array}{l}\text { Dors-P } \\
\text { lantar } \\
\text { Flextio } \\
\text { n }\end{array}$} & No-one & $\begin{array}{l}\text { Young } \\
\text { Older }\end{array}$ & $\begin{array}{l}-1.142 \pm 14.464 \\
-1.959 \pm 14.041 \\
\end{array}$ & $\begin{array}{l}5.51 \pm 30.128 \\
1.988 \pm 5.968\end{array}$ \\
\hline & $\begin{array}{l}\text { One } \\
\text {-both }\end{array}$ & $\begin{array}{l}\text { Young } \\
\text { Older }\end{array}$ & $\begin{array}{l}3.527 \pm 17.194 \\
1.250 \pm 9.9\end{array}$ & $\begin{array}{l}-.433 \pm 2.914 \\
-.768 \pm 3.58\end{array}$ \\
\hline & $\begin{array}{l}\text { No } \\
\text {-both }\end{array}$ & $\begin{array}{l}\text { Young } \\
\text { Older }\end{array}$ & $\begin{array}{l}-4.508 \pm 18.586 \\
-32.79 \pm 115.573\end{array}$ & $\begin{array}{l}1.527 \pm 6.266 \\
18.270 \pm 75.537\end{array}$ \\
\hline \multirow{3}{*}{$\begin{array}{l}\text { Knee } \\
\text { flex- } \\
\text { Extent } \\
\text { ion }\end{array}$} & $\begin{array}{l}\begin{array}{l}\text { No } \\
\text {-both }\end{array} \\
\end{array}$ & $\begin{array}{l}\text { Young } \\
\text { Older }\end{array}$ & $\begin{array}{l}-.92 \pm 4.974 \\
107.85 \pm 432.695\end{array}$ & $\begin{array}{l}.707 \pm 4.369 \\
46.878 \pm 181.522\end{array}$ \\
\hline & $\begin{array}{l}\text { One } \\
\text {-both }\end{array}$ & $\begin{array}{l}\text { Young } \\
\text { Older }\end{array}$ & $\begin{array}{l}.065 \pm 5.061 \\
-.534 \pm 5.233 \\
\end{array}$ & $\begin{array}{l}-.832 \pm 3.516 \\
.91 \pm 3.592\end{array}$ \\
\hline & $\begin{array}{l}\text { No } \\
\text {-both }\end{array}$ & $\begin{array}{l}\text { Young } \\
\text { Older }\end{array}$ & $\begin{array}{l}-5.019 \pm 35.297 \\
5.250 \pm 37.694 \\
\end{array}$ & $\begin{array}{l}-.168 \pm 4.818 \\
49.714 \pm 190.193\end{array}$ \\
\hline \multirow{3}{*}{$\begin{array}{l}\text { Hip } \\
\text { Flex- } \\
\text { Extent } \\
\text { ion }\end{array}$} & No-one & $\begin{array}{l}\text { Young } \\
\text { Older }\end{array}$ & $\begin{array}{l}-.701 \pm 5.529 \\
-36.676 \pm 112.455\end{array}$ & $\begin{array}{l}-3.465 \pm 12.082 \\
17.059 \pm 62.829\end{array}$ \\
\hline & $\begin{array}{l}\text { One } \\
\text {-both }\end{array}$ & $\begin{array}{l}\text { Young } \\
\text { Older }\end{array}$ & $\begin{array}{l}-3.764 \pm 18.52 \\
4.807 \pm 11.917\end{array}$ & $\begin{array}{l}3.117 \pm 15.748 \\
-16.88 \pm 53.259\end{array}$ \\
\hline & $\begin{array}{l}\text { No } \\
\text {-both }\end{array}$ & $\begin{array}{l}\text { Young } \\
\text { Older } \\
\end{array}$ & $\begin{array}{l}26.149 \pm 38.98 \\
31.306 \pm 71.034 \\
\end{array}$ & $\begin{array}{l}-1.647 \pm 10.673 \\
-9.621 \pm 53.889 \\
\end{array}$ \\
\hline \multirow[t]{3}{*}{$\begin{array}{l}\text { Pelvic } \\
\text { Tilt }\end{array}$} & No-one & $\begin{array}{l}\text { Young } \\
\text { Older }\end{array}$ & $\begin{array}{l}13.495 \pm 60.246 \\
28.243 \pm 62.364 \\
\end{array}$ & $\begin{array}{l}17.596 \pm 43.055 \\
253.947 \pm 899.046 \\
\end{array}$ \\
\hline & $\begin{array}{l}\text { One } \\
\text {-both- }\end{array}$ & $\begin{array}{l}\text { Young } \\
\text { Older }\end{array}$ & $\begin{array}{l}26.256 \pm 46.567 \\
5.919 \pm 36.224\end{array}$ & $\begin{array}{l}7.287 \pm 33.334 \\
.791 \pm 38.436\end{array}$ \\
\hline & $\begin{array}{l}\text { No } \\
\text {-both }\end{array}$ & $\begin{array}{l}\text { Young } \\
\text { Older }\end{array}$ & $\begin{array}{l}6.908 \pm 41.463 \\
18.322 \pm 42.859\end{array}$ & $\begin{array}{l}-.19 .878 \pm 50.302 \\
25.267 \pm 48.568\end{array}$ \\
\hline
\end{tabular}

\begin{tabular}{|l|l|l|l|l|}
\hline \multicolumn{2}{|l|}{ Transverse plane } & \multicolumn{2}{c|}{ Movement } \\
\hline & handrail & Age & Ascending & Descending \\
\hline \multirow{4}{*}{ Foot } & No-one & Young & $5.654 \pm 37.093$ & $.479 \pm 16.003$ \\
& & Older & $31.043 \pm 68.215$ & $3.14 \pm 19.822$ \\
\cline { 2 - 5 } Rotaion & One & Young & $3.905 \pm 19.463$ & $5.188 \pm 14.005$ \\
& -both & Older & $-2.431 \pm 9.405$ & $-5.06 \pm 13.248$ \\
\cline { 2 - 5 } & No & Young & $-3.312 \pm 26.698$ & $4.61 \pm 14.655$ \\
& -both & Older & $.604 \pm 30.086$ & $-2.911 \pm 18.278$ \\
\hline Knee & No-one & Young & $.518 \pm 21.407$ & $-12.493 \pm 26.166$ \\
Rotation & & Older & $-6.428 \pm 14.016$ & $1.098 \pm 22.516$ \\
\cline { 2 - 5 } & One & Young & $2.581 \pm 19.731$ & $2.671 \pm 20.563$ \\
& -both & Older & $4.076 \pm 16.998$ & $-4.915 \pm 11.211$ \\
\cline { 2 - 5 } & No & Young & $7.264 \pm 26.867$ & $8.01 \pm 59.984$ \\
& -both & Older & $-10.505 \pm 50.305$ & $68.973 \pm 226.017$ \\
\hline Hip & No-one & Young & $-4.615 \pm 26.67$ & $-.003 \pm 28.083$ \\
Rotaion. & & Older & $2.284 \pm 25.728$ & $-3.356 \pm 18.916$ \\
\cline { 2 - 5 } & One & Young & $-29.484 \pm 34.188$ & $-16.546 \pm 35.028$ \\
& -both* & Older & $2.815 \pm 18.764$ & $-5.018 \pm 19.744$ \\
\cline { 2 - 5 } & No & Young & $-15.369 \pm 58.542$ & $-20.782 \pm 29.559$ \\
& -both & Older & $-.682 \pm 19.504$ & $-7.985 \pm 27.362$ \\
\hline Pelvic & No-one & Young & $23.869 \pm 64.494$ & $-10.847 \pm 33.971$ \\
Rotaion. & & Older & $-2.533 \pm 14.456$ & $27.038 \pm 79.351$ \\
\cline { 2 - 5 } & One & Young & $2.324 \pm 22.432$ & $1.102 \pm 20.38$ \\
& -both & Older & $-1.753 \pm 30.58$ & $-2.833 \pm 39.867$ \\
\cline { 2 - 5 } & No & Young & $.77 \pm 15.294$ & $-14.548 \pm 26.145$ \\
& -both & Older & $-9.006 \pm 26.438$ & $10.252 \pm 53.045$ \\
\hline \multirow{4}{*}{ p<.05 } & \multicolumn{3}{|c}{} \\
\hline
\end{tabular}

During stair ascent young and older adults were found to generate similar joint movement but knee and hip joint movements were distributed differently(fig.1) The concept of joint movement being distributed differently between young and older adults(Devita and Hortobagyi, 2000). The older adults reduced the joint movement at the knee but increased the hip movement compared to young adults.

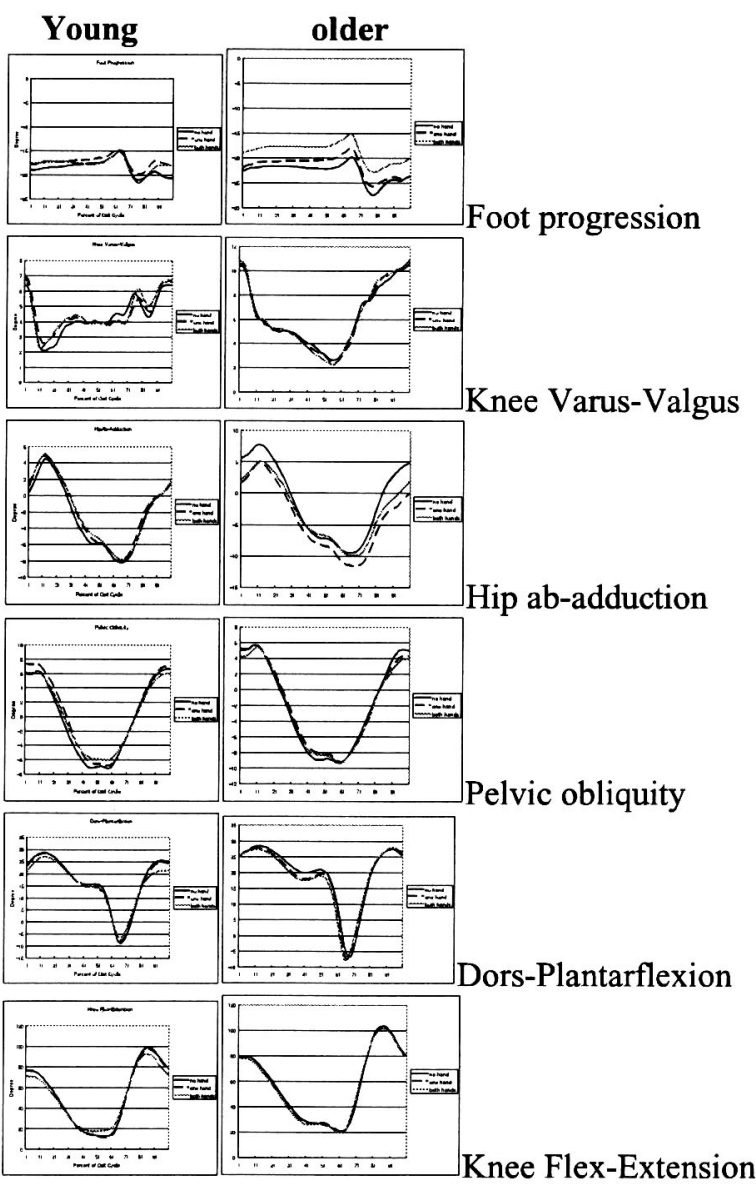




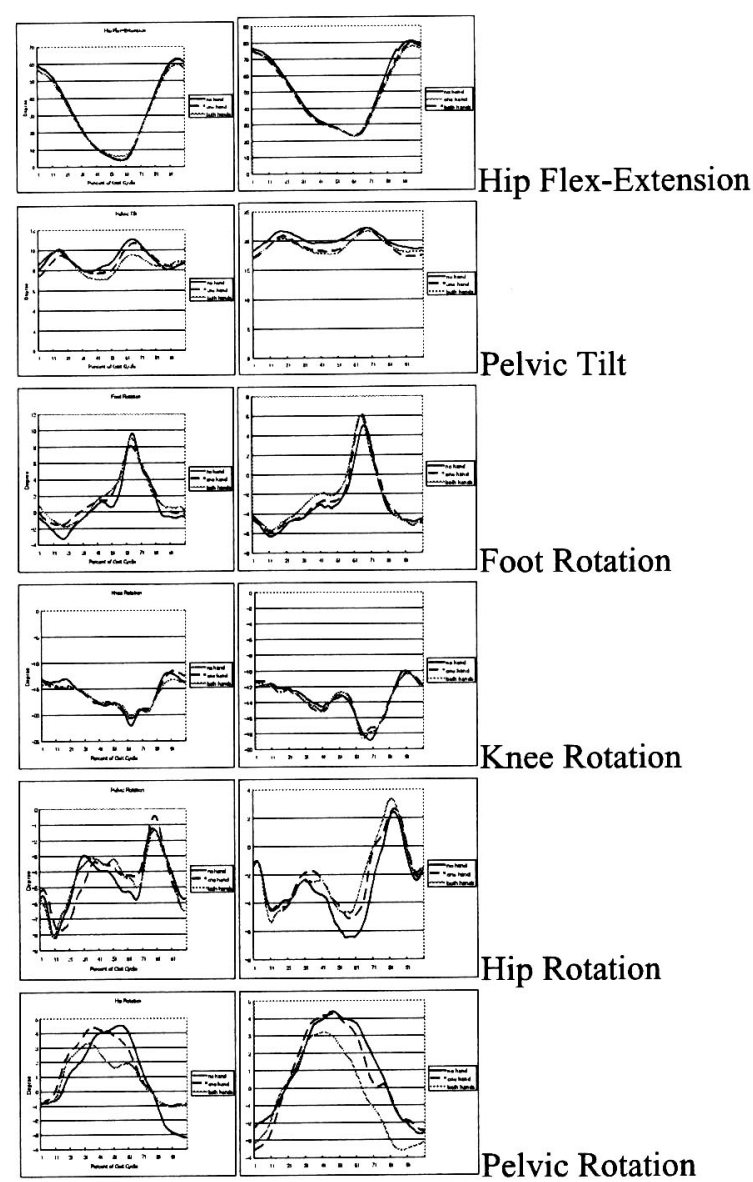

Fig. 1. Joint kinematic demands of stair ascent in young and old adults

During stair descent the knee extension-flexion and ankle dorsiflexion - Plantarflexion angle changes were similar between young and older adults (fig. 2). But foot, knee, hip and pelvic rotation were distributed differently in the stride cycle. Observations of sagittal plane motion older adults include decreased plantarflextion and hip-flex extension at contact relative to young adults. These observations are related to shorten stride in order adults.

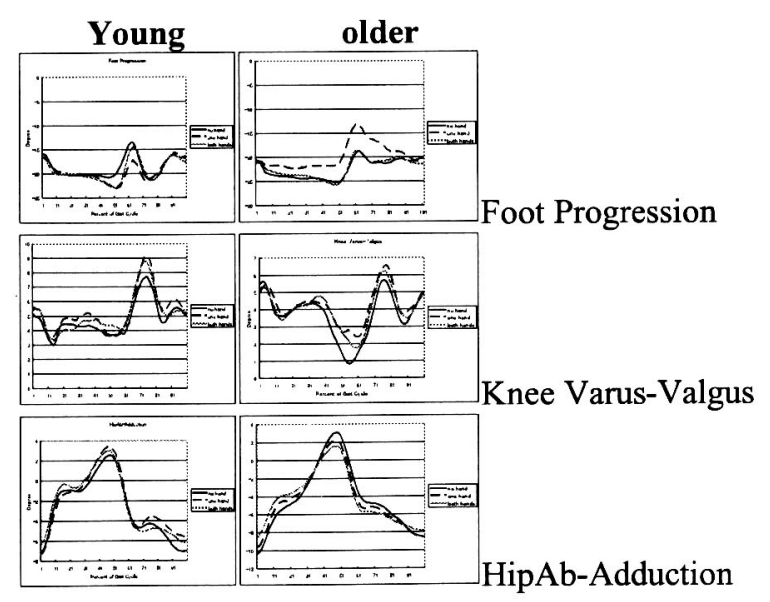

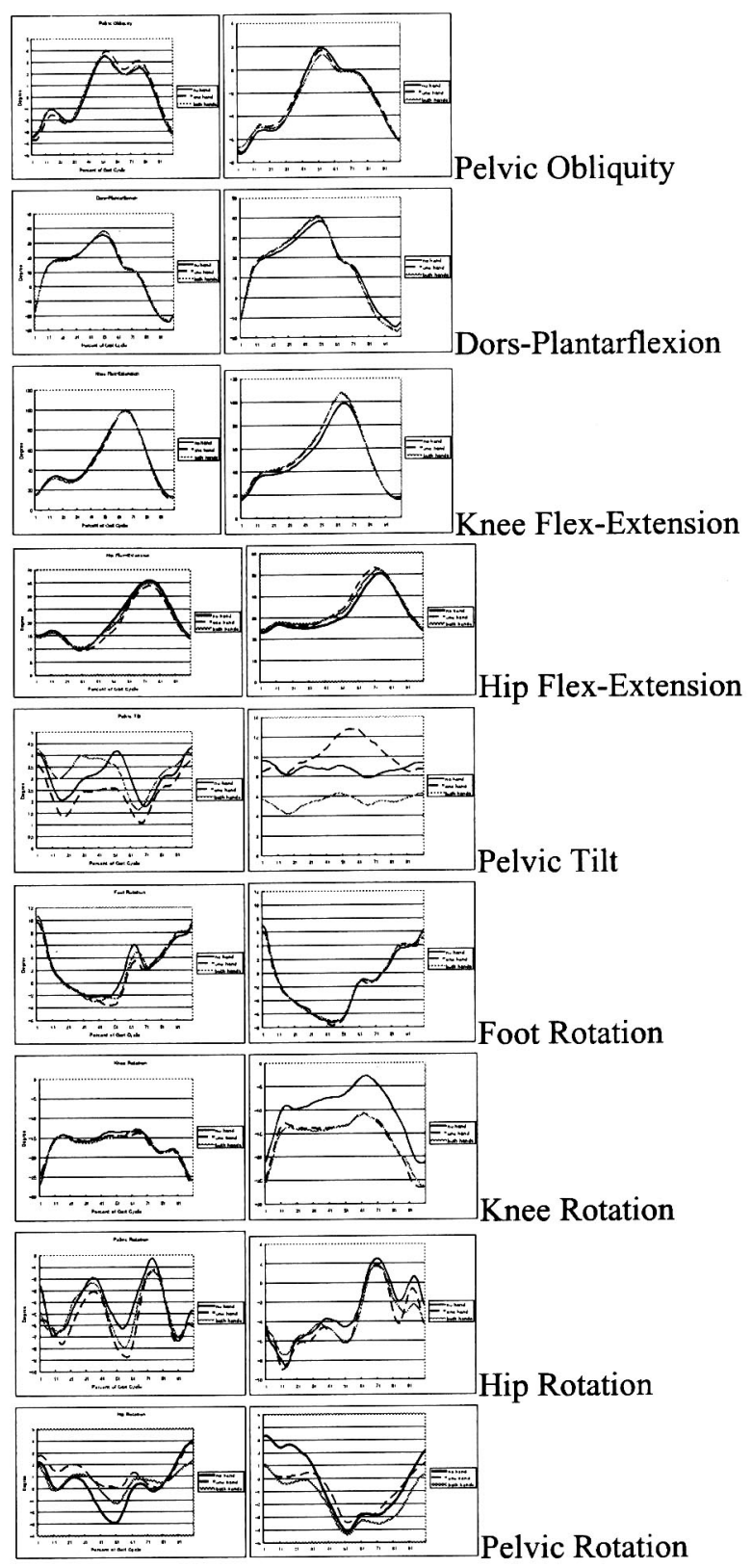

Fig. 2. Joint kinematic demands of stair descent in young and older adults

\section{References}

[1] Anastasia. P, Wendy I. Drechlsler, Mary C. Cramp, Fiona J. Coutts, Oona M. Scott, "Hip, Knee, ankle kinematics and kinetics during stair ascent and descent in healthy young individuals", Clinical Biomechanics 22 pp.203-210, 2007.

[2].Devita P, Hortobagyi T, Age cause a redistribution of joint torques and powers during gait. J Appl Physiol, 88, 1804-1811, 2000.

[3] Flynn, J., "Kinematical variability of normal climbing and descending stairs." M.Sc. Thesis, Vanderbilt University, Nashville, TN, 1977.

[4] Hortobagyi, T., Mizelle C, DeVita P., Old adults perform activities of daily living near their maximal capabilities. Journal Geronto; A: boil Sci Med Sci 58, M 453-460. 2003. 
[5] McFadyen, B. J., Winter, D. A., "An integrated biomechanical analysis of normal stair ascent and descent", Journal of Biomechanics.21, pp.733-744,1988. [6] Robert Riener, Marco Rabuffetti, Carlo Frigo, "Stair ascent and descent at different inclinations", Gait Posture, pp.32-44, 2002.

[7]. Maki, B.E., Perry, S.D., Mcllroy, W.E. "Efficacy of handrails in preventing stairway falls", Safety Science Vol.28, No.3, pp.189-206,1998

[8] Nadeau, S., McFadyen, B.J., Malouin, F., "Frontal and sagittal plane analyses of the stair climbing task in healthy adults agerd over 40 years", Clinical Biomechanics, pp950-959, 2003.

[9] Seon-Deok Eun, Yeon-Joo Yu, Chong-Hoon, Lee, The effect of the stair height on the gait pattern in stair-descent activity of elderly persons, Journal of sport and leisure studies, , Vol.29, pp.443-451,2007.

[10] Wansoo lim, Taebeum Ryu, Hoonwoo choi, Hwa Soon Choi,, Min k. Chung, A comparison of gait characteristics between Korean and western youn people, journal of rgonomics society of korea, vol 25 , no. 2 , pp.33-41,May 2006. 